\title{
A modelação do jogo em Futsal. Análise sequencial do $1 \times 1$ no processo ofensivo
}

\author{
Rui Amaral \\ Júlio Garganta
}

https://doi.org/10.5628/rpcd.05.03.298

\author{
Universidade do Porto \\ Faculdade de Desporto \\ Portugal
}

\section{RESUMO}

As interrogações clássicas dos jogos desportivos colectivos, $o$ quê, quando, como e porque fazer, adquirem na situação de $1 \times 1 \mathrm{em}$ Futsal uma importância crucial, em virtude das características específicas desta modalidade. No presente artigo procura-se: (i) identificar as acções táctico -técnicas que são induzidas (activadas) pelo $1 \mathrm{x} 1$, e as que são indutoras (activadoras) da referida situação; (ii) descrever as características das situações de $1 \times 1$ que mais frequentemente induzem o desequilíbrio defensivo do adversário; (iii) fornecer, a partir dos resultados obtidos, algumas orientações práticas para o treino do $1 \times 1$. A amostra do estudo foi constituída por cinco jogos do campeonato nacional da primeira divisão, tendo -se registado 853 sequências e 8559 ocorrências. Em termos metodológicos foi utilizada a metodologia obser vacional, mais concretamente, a análise sequencial e a técnica de coordenadas polares. Recorreu-se ao software SDISGSEQ para o registo e tratamento dos dados. A partir dos resultados obtidos conclui-se que: prospectivamente, o $1 \mathrm{x} 1$ parece fomentar o desequilíbrio defensivo do adversário, uma vez que é activador de situações de falta e de remate; retrospectivamente, o $1 \mathrm{x} 1$ parece ser activado por condutas de condução de bola, combinação táctica com progressão e recuperação de bola; à medida que o $1 \mathrm{x} 1$ é realizado numa zona mais ofensiva do campo, parece aumentar a probabilidade de este originar um desequilíbrio na estrutura defensiva adversária; o drible de progressão e o drible para remate são os tipos de $1 \mathrm{x} 1$ que mais provocam o desequilíbrio defensivo do adversário; ao nível do conte xto de cooperação, o $1 \times 1$ com vários apoios é aquele que apresenta maior probabilidade de conduzir a uma situação de remate.

Palavras-chave: futsal, 1x1, análise do jogo, metodologia observacional, análise sequencial.

\section{ABSTRACT \\ Game modelling in futsal. Sequential analysis of $1 \times 1$ in attacking process.}

The classic questions of team sports, what, when, who and why do it, have a vital importance in Futsal 1 on 1 situations, due to the specific characteristics of this sport. In this paper it is intended: (i) to identify tactical-technique actions that induce or are induced by 1 on 1; (ii) to describe the characteristics of 1 on 1 situations that more often promote the rupture in opponent defensive organization; (iii) to provide some practical orientations for 1 on 1 training. The sample includes 853 sequences and 8559 conducts, registered from the observation of five matches of the National First League. Observational methodology was used, with special reference to the sequential analysis and polar coordinates technique. For the treatment of data SDIS-GSEQ software was used. From the results we conclude that: prospectively, the 1 on 1 seems to encourage opponent defensive rupture, since it activates fouls and shots at goal situations; retrospectively, 1 on 1 seems to be activated by ball conduction, tactical combination with progression in the field and ball recovery; the possibility of defensive rupture in the opposing team grows as 1 on 1 situation takes place closer to the opponent goal; dribble of progression and dribble for shot at goal, are the dribbling types that most induce defensive ruptures; in respect to the cooperation context, the 1 on 1 with various supports (pass lines) presents a larger possibility of originating a shot on goal.

Key Words: futsal, 1 on 1, match analysis, observational methodology, sequential analysis. 


\section{INTRODUÇÃO}

A modalidade de Futsal, nascida durante a década de 30 na América do Sul (Brasil ou Uruguai) (26, 28, 31 ), tem evidenciado desde os anos 90 um claro desenvolvimento à escala mundial.

Actualmente, fruto da evolução dos regulamentos, da especialização da modalidade em termos técnicos, tácticos e energético -funcionais, bem como da proveniência dos jogadores dos escalões de formação, o Futsal parece afastar -se cada vez mais da modalidade que lhe deu origem - o Futebol - conquistando um espaço próprio no universo dos jogos desportivos colectivos (JDC).

Não obstante o claro desenvolvimento, a investigação nesta modalidade é ainda escassa. Como podemos verificar no quadro 1, grande parte dos trabalhos realizados tem incidido fundamentalmente sobre os aspectos táctico -técnicos e energético -funcionais, constatando -se um aumento recente do interesse pelos primeiros.

Quadro 1. Trabalhos sobre Futsal encontrados na presente pesquisa.

Exigências energético-funcionais.

Molina, 1992 (19); D’Ottavio, 199 ? (8); Oliveira, 1998 (21);

Facchin et al., 1999 (11); Moreno, 2001 (20); Álvarez et al., 2002 (3).

Aspectos tácticos e técnicos.

Sannicandro, 1995 (27); Oliveira, 1998 (21); Facchin et al., 1999 (11); Mendes, 2002 (16); Matos, 2002 (15); Silva, 2002 (30); Abreu, 2002 (1); Silva Matos, 2002 (29);

Canastra, 2002 (?); Fernandes, 2003 (12).

O perfil genético, antropométrico, de somatótipo e de aptidão física dos jogadores.

Duarte, 1988 (10); Dantas e Filho, 2002 (9)

A capacidade de tomada de decisão e o conhecimento declarativo.

Souza, 2002 (31)

\section{A importância da situação de jogo $5 \times 5$ para a formação e treino em Futebol.}

Allen et al., 1998 (2); Bezerra, 1999 (5); Platt et al., 2001

(25); Hoff et al., 2002 (14)
Este quadro ilustra o facto de estarmos em presença de uma modalidade que carece de investigação em vários domínios, sendo um deles a vertente táctico -técnica. Entre os aspectos táctico -técnicos, a situação de $1 \times 1$, quer do ponto de vista ofensivo, quer do ponto de vista defensivo, é uma das que mais tem estimulado a nossa refle xão sobre o jogo.

As interrogações clássicas dos JDC, o quê, quando, como e porque fazer (13), às quais acrescentamos o onde fazer, adquirem na situação de 1x1 em Futsal uma clara importância, em virtude das características específicas desta modalidade.

É nossa convicção que, através do $1 \times 1$ no Futsal, uma equipa pode rapidamente provocar o desequilíbrio defensivo do adversário no caso do $1 \mathrm{x} 1$ ser bem sucedido, mas, em contrapartida, pode também ela ver-se desequilibrada em termos defensivos, se o $1 x 1$ conduzir à recuperação de bola por parte do adversário. Por este motivo trata-se de uma situação crítica do jogo, devendo merecer especial atenção por parte de treinadores e investigadores.

Com o presente estudo procurou-se: (i) identificar as acções táctico -técnicas que são induzidas (activadas) pelo $1 \mathrm{x} 1$, e as que são indutoras (activadoras) da referida situação; (ii) descrever as características das situações de $1 \mathrm{x} 1$ que mais frequentemente provocam $\mathrm{o}$ desequilíbrio defensivo do adversário; (iii) fornecer algumas orientações práticas para o treino do $1 \times 1$.

\section{METODOLOGIA}

\section{A metodologia observacional}

A metodologia obser vacional é uma das opções de estudo científico do comportamento humano (4), revelando -se uma estratégia de investigação apropriada para analisar a acção motora nos JDC (24). Tem por objecto de estudo o indivíduo inserido em qualquer um dos seus conte xtos habituais de actuação, neste caso específico o desporto (4). Esta metodologia requer o cumprimento de alguns requisitos básicos (4), a saber: a espontaneidade do comportamento, não devendo o investigador condicionar a actuação dos sujeitos; a obser vação realizada em conte xto natural, o mesmo será dizer em situação de jogo; a elaboração de instrumentos ad hoc, consistindo este requisito na construção de sistemas de categorias que se adaptem à realidade prática e ao enquadramento teórico do assunto que pretendemos estudar; e continuidade temporal. 


\section{A análise sequencial}

O termo análise sequencial refere-se a um conjunto de técnicas que têm como objectivo evidenciar as relações, associações e dependências sequenciais entre unidades de conduta. Este tipo de análise consiste em averiguar as probabilidades de ocorrência de determinadas condutas, em função da prévia ocorrência de outras (17). Como meta procura-se a comprovação de uma ordem sequencial, isto é, uma certa estabilidade na sucessão de sequências, que se encontre acima das probabilidades que são explicáveis pelo acaso (18).

$\mathrm{Na}$ análise sequencial deve considerar -se dois tipos de conduta (6): a critério (CC), que é a categoria a partir da qual, na sequência de dados, se contabilizam as transições, ou retardos, de forma prospectiva (para a frente), ou retrospectiva (para trás); e a objecto (CO), que é a categoria até onde, na sequência de dados, se contabilizam as transições/ retardos. A análise pode ter um carácter prospectivo (análise da sequência de condutas que se seguiram à conduta critério: $1,2, \ldots$ ), ou retrospectivo (análise da sequência de condutas que antecederam a conduta critério: $-1,-2, \ldots)(23)$.

No presente trabalho pretendemos utilizar as duas perspectivas de análise até aos retardos 5 e -5 .

\section{A análise pela técnica de coordenadas polares}

No conte xto da análise do jogo, a técnica de coordenadas polares, devido à sua grande capacidade de síntese em relação aos dados, permite (22): a leitura da relação entre as várias condutas de jogo desenvolvidas pelas equipas; a detecção do grau de afinidade entre condutas; a constatação de relações de activação ou inibição entre condutas, quer prospectivamente, quer retrospectivamente.

Como tal, esta técnica é indicada em conte xtos de grande comple xidade, onde se verificam múltiplas relações entre as várias categorias do sistema de obser vação (17).

\section{Instrumento de observação}

O instrumento utilizado no presente estudo teve por base o sistema de categorias construído por Caldeira (6) para a análise do $1 \times 1$ no processo ofensivo em Futebol, tendo sido sujeito às necessárias alterações, em virtude da especificidade do Futsal.

O sistema de categorias é um dos instrumentos básicos da metodologia obser vacional. Sendo elaborado pelo obser vador com base na realidade empírica e no suporte teórico, caracteriza-se por ser um sistema fechado, de codificação única, e não autorregulável. Para a adaptação do instrumento à especificidade do Futsal, contámos com a bibliografia específica da modalidade, trabalhos realizados com recurso à análise sequencial no âmbito dos JDC e sessões de obser vação exploratórias, que visaram melhorar o sistema de categorias, bem como a própria metodologia de obser vação.

No quadro 2 pode obser var-se o sistema de categorias configurado para o presente estudo. 
Quadro 2. Sistema de categorias (conduta e respectivo código).

\begin{tabular}{|c|c|c|}
\hline \multicolumn{2}{|c|}{ Macrocategorias } & Categorias \\
\hline \multicolumn{2}{|c|}{$\begin{array}{l}\text { Macrocategoria } 1 \\
\text { Início do Processo Ofensivo }\end{array}$} & $\begin{array}{l}\text { Pontapé de saída (PS); Lançamento de baliza (LB); Pontapé de linha lateral } \\
\text { (RPLL); Recuperação directa por intercepção (RDI), desarme (RDD) ou duelo } \\
\text { (RDDU); Recuperação de bola indirecta RBI (RBI); Bola ao solo (BS) }\end{array}$ \\
\hline \multicolumn{2}{|c|}{$\begin{array}{l}\text { Macrocategoria } 2 \\
\text { Construção e Desenvolvimento } \\
\text { do Processo Ofensivo }\end{array}$} & $\begin{array}{l}\text { 1×1 (1×1); Condução de bola (CB); Combinação táctica sem progressão (CTS), } \\
\text { com progressão (CTP), com movimento da bola contrário ao sentido de ataque } \\
\text { (CTR) ou directa (CTD); Tentativa de passe (TP); Pontapé de linha lateral (PLL) } \\
\text { ou de canto (PC); Falta (FAL); Cruzamento (CRUZ); Intervenção do adversário } \\
\text { (IAD) ou do guarda-redes adversário (IGR); Remate interceptado pela defesa } \\
\text { (RI); Remate enquadrado (RE); Duelo (DUEL) }\end{array}$ \\
\hline \multicolumn{2}{|c|}{$\begin{array}{l}\text { Macrocategoria } 3 \\
\text { Finalização do Processo Ofensivo }\end{array}$} & $\begin{array}{l}\text { Perda de bola directa (PBD) ou indirecta (PBI); remate não enquadrado (RNE); } \\
\text { Golo (GOLO) }\end{array}$ \\
\hline \multirow{3}{*}{$\begin{array}{l}\text { Subcategorias do } \\
\qquad 1 \times 1\end{array}$} & Zona de Ocorrência & Zona 1 (D1), 2 (D2), 3 (D3) ou 4 (D4) \\
\hline & Tipo de $1 \times 1$ & $\begin{array}{c}\text { Drible de progressão (DPRG), protecção (DPRT), para passe (DPSS) ou para } \\
\text { remate (DREM] }\end{array}$ \\
\hline & $\begin{array}{l}\text { Contexto de } \\
\text { Cooperação }\end{array}$ & Sem apoios (DSA), com um apoio (DUA) ou com dois ou mais apoios (DVA) \\
\hline
\end{tabular}

O instrumento de obser vação foi organizado em categorias exaustivas e mutuamente exclusivas (E/ME). Tal implicou que qualquer comportamento que se encontrasse dentro do âmbito considerado como objecto de estudo pudesse ser sempre registado em uma das categorias (exaustividade), e cada comportamento dos anteriormente referidos apenas pudesse ser assinalado em uma, e só uma, das categorias (mútua exclusividade) (4).

\section{Amostra}

Para a realização do presente estudo foram obser vados cinco jogos do Campeonato Nacional da $1^{\text {a }}$ divisão, transmitidos pela televisão, e relativos à época desportiva 2003-2004. Os jogos obser vados foram os seguintes:

A. R. Freixieiro - S. L. Benfica

Boavista F. C. - G. D. Fundação Jorge Antunes

S. L. Benfica - Famalicense

S. L. Benfica - Sporting C. P.

A. R. Freixieiro - Sporting C. P.

A amostra foi constituída por todos os processos ofensivos que permitiam obser var a totalidade das condutas que deles fizeram parte. Nos casos em que não foi possível obser var todas as condutas por ine- rência da transmissão televisiva (repetição de uma jogada anterior, corte na transmissão ou filmagem de um local diferente daquele onde se encontra a bola), optou-se por eliminar toda a sequência.

No total, a amostra do presente estudo engloba 853 sequências ofensivas, a partir das quais se registaram 8559 ocorrências.

\section{Material}

Para a visualização das imagens foi utilizada uma televisão PHILIPS modelo 21PT136B. Para a gravação e reprodução dos jogos recorreu-se a um vídeogravador SONY do modelo SLV-E100, sistema VHS. Para o registo e tratamento dos dados usou-se um computador portátil com processador pentium IV (1.80 GHz, $228 \mathrm{~KB}$ de RAM), equipado com o software SDIS (Sequential Data Interchange Standard)GSEQ (Generalized Sequential Querier) 4.1 (versão para windows).

\section{Procedimentos de observação}

O registo dos dados foi realizado a posteriori, através da visualização das filmagens televisivas dos jogos. Com o objectivo de registar correctamente todos os comportamentos do processo ofensivo, nomeadamente as características das situações de $1 \times 1$, adop- 
támos a estratégia de obser var cada sequência ofensiva tantas vezes quantas as necessárias, em velocidade normal ou utilizando a função de "pause". Para a notação dos dados da obser vação utilizou-se um arquivo com extensão SDS, no qual foram registados os códigos dos comportamentos obser vados. Cada sequência ocupou uma linha terminada com ponto e vírgula, à excepção da última que terminou com uma barra inclinada (/), indicadora do fim da unidade de análise. O início de um novo processo ofensivo implicou a passagem para a linha seguinte. Esta forma de registo implicou que cada sequência correspondesse a uma sessão na linguagem do software SDIS-GSEQ, de modo a que os dados de determinada sequência ofensiva não pudessem ser contabilizados nas sequências adjacentes.

A título de exemplo, é apresentada no quadro 3 a forma como algumas das sequências ofensivas que resultaram em golo foram registadas no programa SDIS- GSEQ.

Quadro 3. Registo, no SDIS-GSEQ, de algumas das sequências ofensivas que terminaram em golo.

\begin{tabular}{|l|l|}
\hline JOG0 & SEQUÊNCIA \\
\hline Freixieiro - Benfica & RDI3 CB3 D4SU CRUZ RE4 GOLO; \\
\hline Boavista - Fundação J. A. & RPLL4 CTR4 D4RV RE4 GOLO; \\
\hline Benfica - Famalicense & RDI1 CTP1 CTR3 D3PU CB3 CTP3 CB4 FAL4 RE4 GOL0; \\
\hline Benfica - Sporting & RDI4 RE4 GOL0; \\
\hline Freixieiro - Sporting & RDI2 CB2 D2PU CTP2 CB3 D3RV RE3 GOL0; \\
\hline
\end{tabular}

Na figura 1, estão representadas as zonas do campograma utilizadas como referência para espacialização das acções de $1 \mathrm{x} 1$ no presente estudo.

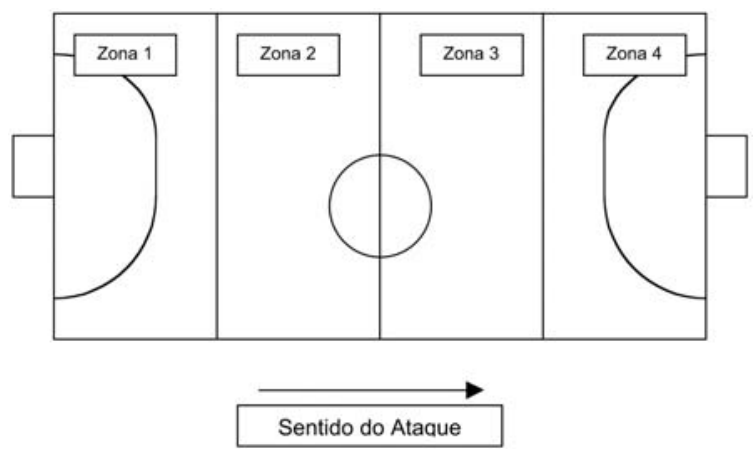

Figura 1. Campograma relativo à espacialização das acções de $1 \times 1$.

\section{ANÁLISE E DISCUSSÃO DOS RESULTADOS}

\section{Análise descritiva}

No presente estudo foram registadas 730 situações de $1 \times 1$, as quais representam $8,5 \%$ do total de con- dutas ocorridas. Estes valores revelam uma elevada frequência das acções de $1 \times 1$, facto que aumenta a necessidade de melhor se conhecer a importância desta estrutura de jogo no Futsal.

Relativamente à zona do campo, verificou-se que a maior parte das situações de $1 \times 1$ ocorreu na zona 3 $(42,6 \%)$, enquanto a zona 1 é aquela onde menos vezes o $1 x 1$ é levado a cabo (13,15\%). As zonas 2 e 4 apresentam valores intermédios, com $21,78 \%$ e $22,47 \%$, respectivamente. A explicação para a baixa percentagem de situações de $1 \times 1$ na zona 1 , pode ter a ver com o elevado perigo que uma perda de bola nesta zona pode significar, o que leva a que os jogadores optem por soluções tácticas menos arriscadas. Por outro lado, a zona 3 encontra-se já no meio campo ofensivo, onde a relação risco/benefício do $1 \mathrm{x} 1$ é mais favorável à equipa atacante.

A partir da análise do quadro 4 constata-se que o tipo de drible mais utilizado é o de progressão $(46,71 \%)$. Este facto parece indiciar que os jogadores de Futsal entram em situação de $1 x 1$ fundamentalmente com a intenção de ultrapassar o seu adversário directo e progredir no terreno de jogo. 
Quadro 4. Frequência relativa das situações de $1 \times 1$, atendendo à zona, tipo e contexto de cooperação.

\begin{tabular}{|c|c|c|c|c|c|c|c|c|c|c|c|c|c|}
\hline & \multicolumn{3}{|c|}{ ZONA 1} & \multicolumn{3}{|c|}{ ZONA 2} & \multicolumn{3}{|c|}{ ZONA 3} & \multicolumn{3}{|c|}{ ZONA 4} & \\
\hline & \multicolumn{3}{|c|}{$13.2 \%$} & \multicolumn{3}{|c|}{$21.8 \%$} & \multicolumn{3}{|c|}{$42.6 \%$} & \multicolumn{3}{|c|}{$22.5 \%$} & \\
\hline & $S$ & U & V & $S$ & U & V & $S$ & U & V & $S$ & U & V & TOTAL \\
\hline PROG & 1.10 & 0.82 & 2.33 & 3.01 & 4.11 & 6.58 & 7.26 & 6.44 & 7.53 & 4.52 & 1.78 & 1.23 & $46.71 \%$ \\
\hline PROT & 1.10 & 1.78 & 1.37 & 0.68 & 2.60 & 1.92 & 2.05 & 3.15 & 3.97 & 3.01 & 1.92 & 0.82 & $24.38 \%$ \\
\hline REM & . & . & . & - & . & . & 0.41 & 1.10 & 5.34 & 1.10 & 1.78 & 1.64 & $11.37 \%$ \\
\hline PAS & 0.41 & 1.64 & 2.60 & 0.03 & 0.82 & 1.78 & 1.51 & 0.96 & 2.88 & 1.92 & 2.19 & 0.55 & $17.53 \%$ \\
\hline TOTAL & 2.60 & 4.25 & 6.30 & 3.97 & 7.53 & 10.27 & 11.23 & 11.64 & 19.73 & 10.55 & 7.53 & 4.25 & $100 \%$ \\
\hline
\end{tabular}

\begin{tabular}{l|l|l|l|} 
TOTAL & $\mathbf{2 8 . 3 6}$ & $\mathbf{3 1 . 1 0}$ & $\mathbf{4 0 . 5 5}$ \\
\hline
\end{tabular}

Por seu lado, o drible para remate é aquele que apresenta menor frequência $(11,37 \%)$, facto que não é de estranhar, visto que este tipo de $1 \mathrm{x} 1$ só faz sentido a partir de um determinado ponto do terreno, normalmente os últimos quinze metros do campo. Por fim, surgem os tipos drible de protecção e drible para passe com, respectivamente, $24,38 \%$ e $17,53 \%$ do total das acções de $1 \times 1$. Daqui devemos salientar o facto de que em cerca de um quarto das situações de 1x1 o jogador apenas ter como intenção proteger a posse da bola.

Relativamente ao conte xto de cooperação é interessante obser var que em $40,55 \%$ das situações de $1 \times 1$, o jogador de posse da bola dispunha de dois ou mais apoios, isto é, de linhas de passe. Para além disso, em $31,10 \%$ das acções de 1x1 levadas a cabo, o portador da bola dispunha de um apoio. E só em 28,36\% das situações o jogador de posse da bola não possuía nenhuma linha de passe quando optou por jogar o 1x1. Assim, somos levados a concluir que a ausência de linhas de passe não é um factor determinante para os jogadores optarem por jogar o $1 \mathrm{x} 1$.

Paradoxalmente, a opção por jogar $1 \mathrm{xl}$ parece surgir com maior frequência quando o portador da bola possui maior número de linhas de passe, talvez pelo facto de, nesse conte xto, tal acção se afigurar menos provável e, portanto, constituir um factor de surpresa.

\section{Análise sequencial}

No quadro 5 encontra-se representada a análise sequencial prospectiva até ao retardo 5 , tendo o $1 \times 1$ como conduta critério.

Quadro 5. Análise sequencial prospectiva até ao retardo 5, para as situações de 1x1.

\begin{tabular}{|c|c|c|c|c|c|}
\hline CC & 1 & 2 & 3 & 4 & 5 \\
\hline $1 \times 1$ & $\begin{array}{l}\text { FAL [12.42] } \\
\text { REM [10.82] } \\
\text { IAD [9.88] } \\
\operatorname{PLL}[5.87] \\
\operatorname{PBD}[5.07] \\
\operatorname{TP}[4.99] \\
\text { CRUZ [3.71] } \\
\text { DUEL [2.28] } \\
\text { LB }[2.00]\end{array}$ & $\begin{array}{l}\text { PBD [9.79] } \\
\text { PC [4.54] } \\
\text { PLL [4.31] } \\
\text { IAD [3.22] } \\
\text { DUEL (2.59] } \\
\text { REM [2.53] }\end{array}$ & $\begin{array}{l}\text { PC }[3.32] \\
\text { CRUZ }[2.58] \\
\text { PBD }(2.06)\end{array}$ & IGR [3.02] & $P C(2.89)$ \\
\hline & $p=0.0000$ & $p=0.0000$ & $p=0.000446$ & $p=n s$ & $p=0.048405$ \\
\hline
\end{tabular}


Neste quadro podemos obser var os resíduos ajustados z (valores colocados entre parêntesis), que nos irão permitir conhecer as condutas do sistema de categorias que apresentam uma probabilidade de ocorrência, após o $1 \times 1$, acima do que seria de esperar enquanto fruto do acaso.

Os resultados mostram que as acções de $1 \mathrm{x} 1$ influenciam de forma determinante o desenvolvimento do jogo de Futsal. Assim, verifica-se que as situações de falta apresentam uma elevada probabilidade de serem activadas pelas acções de $1 \times 1$, o que no Futsal, em virtude das regras de acumulação de faltas e dos livres directos sem barreira, assume uma enorme importância.

Existe igualmente uma elevada probabilidade do 1x1 activar uma situação de remate, o que significa que muitas vezes a nossa CC, por si só, permite ao ataque chegar à sua última fase: a finalização. Salientese ainda que existe também a probabilidade, embora menor que na transição 1 , de após uma acção de $1 \times 1$ ocorrer um remate ao nível da transição 2 .

Verificámos ainda uma elevada probabilidade do $1 \times 1$ activar a conduta de inter venção do adversário, embora sem que este consiga recuperar a bola com essa primeira acção.
Um dos aspectos mais salientes desta análise é que existe uma grande probabilidade das situações de $1 x 1$ promoverem a ruptura do jogo, isto é, a saída da bola do terreno (PLL, LB, PC). Esta probabilidade é elevada para todas as transições consideradas. O risco de perda da bola é também uma probabilidade a ser considerada quando o jogador de Futsal opta por uma situação de $1 x 1$, colocando -se esta possibilidade fundamentalmente ao nível das três primeiras transições, e com especial incidência na segunda.

Após o $1 x 1$ é também provável que o jogador tente imediatamente efectuar um passe que não chegue ao receptor (TP). Os cruzamentos e os duelos apresentam uma probabilidade de ocorrência superior ao que seria de esperar após uma acção $1 \times 1$.

Os valores obtidos ao nível da transição 4 não são analisados, em virtude do p obtido para esta transição não se ter revelado significativo, uma vez que apresenta um valor superior a 0.05 (estatística Chi Quadrado de Pearson). Para a análise retrospectiva ao $1 \mathrm{x} 1$ seguimos o mesmo processo que para a análise prospectiva (quadro 6)

Quadro 6. Análise sequencial retrospectiva até ao retardo -5 tendo o 1x1 como conduta critério.

\begin{tabular}{|c|c|c|c|c|c|}
\hline .5 & -4 & .3 & -2 & -1 & CC \\
\hline $\begin{array}{l}\text { RDI }(2.79] \\
\text { FAL }[2.35] \\
\text { PS [2.01] }\end{array}$ & $\begin{array}{l}\operatorname{CTR}[2.16] \\
\operatorname{REM}(2.13]\end{array}$ & RDI [2.39] & $\begin{array}{l}\text { RDI [?.74) } \\
\text { CTP }[3.44] \\
\operatorname{RDD}(1.96)\end{array}$ & $\begin{array}{l}\text { CB (15.28) } \\
\text { СTP }[8.39]\end{array}$ & \multirow[t]{2}{*}{$1 \times 1$} \\
\hline$p=0.048023$ & $\mathrm{p}=\mathrm{ns}$ & $\mathrm{p}=\mathrm{ns}$ & $p=0.000000$ & $p=0.000000$ & \\
\hline
\end{tabular}

Retrospectivamente destaca-se o elevado resíduo ajustado obtido pelas condutas de combinação táctica com progressão (CTP) e condução de bola (CB) ao nível do retardo -1 .

Estes resultados parecem compatibilizar -se com a lógica do jogo, uma vez que a condução de bola (CB) e a combinação táctica com progressão (CTP) são condutas que implicam a progressão da bola no terreno de jogo. A esta progressão a defesa deve responder com uma maior oposição, materializada, por exemplo, numa contenção defensiva mais agressiva, a qual o portador da bola pode perfeitamente tentar ultrapassar através de uma acção de $1 \times 1$.

Por outro lado, o facto do $1 \mathrm{x} 1$ ter sido activado, a duas transições de distância, por acções de recuperação de bola, quer por intercepção (RDI), quer por desarme (RDD), e por combinações tácticas com progressão (CTP), parece sugerir que o $1 \times 1$ foi um meio muito utilizado pelos jogadores em situações de rápida transição defesa-ataque ou contra-ataque, uma vez que esta acção foi estimulada retrospectivamente por acções de recuperação da bola (RDI e RDD), de jogo 
mais directo e objectivo (CTP), e de condução da bola (CB).

Após analisar os dados pela técnica de retardos, os mesmos foram submetidos à análise pela técnica de coordenadas polares, através da qual se pode verificar o tipo de relação (activação ou inibição) que o $1 x 1$, enquanto conduta critério, estabelece com as restantes condutas do sistema de obser vação (figura 2).

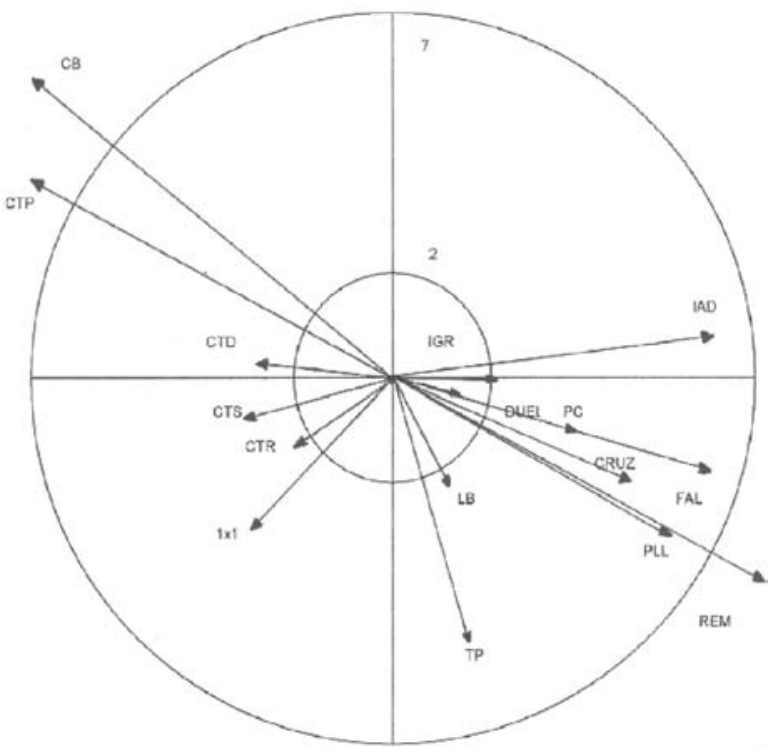

Figura 2. Mapa de coordenadas polares, tendo o 1x1 como conduta critério.

A análise do mapa de coordenadas polares permite verificar que as acções de $1 \times 1$ activam e são activadas pela conduta inter venção do adversário (IAD), dado que o vector representativo desta conduta se encontra no quadrante 1 .

Pelo contrário, os vectores que se encontram traçados no quadrante 3 , significam que a relação entre o 1x1 e essas condutas é de mútua inibição, isto é, retrospectivamente essas condutas inibem o aparecimento do $1 \mathrm{x} 1$, e prospectivamente o $1 \mathrm{x} 1$ inibe $\mathrm{o}$ aparecimento destas condutas. Nesta situação encontram-se as categorias de $1 \times 1$, combinação táctica sem progressão (CTS) e combinação táctica com retrocesso (CTR).
No quadrante 2 estão representadas as condutas que activam retrospectivamente $\mathrm{o} 1 \mathrm{x} 1$, mas são inibidas por este em termos prospectivos. Neste quadrante surgem os vectores representativos das condutas condução de bola (CB), combinação táctica com progressão (CTP) e combinação táctica directa (CTD). Por fim, no quadrante 4, encontram-se representados os vectores das condutas que prospectivamente são activados pelo $1 \times 1$, mas que retrospectivamente o inibem. Na presente análise é neste quadrante que se encontram o maior número de condutas, a saber, o remate (REM), a falta (FAL), o pontapé de linha lateral (PLL), a tentativa de passe (TP), o cruzamento (CRU Z), o pontapé de canto (PC), o lançamento de baliza (LB), a inter venção do guarda-redes (IGR) e o duelo (DUEL).

O tamanho do vector representa a «intensidade» da relação, logo, deve salientar -se o facto do $1 \mathrm{x} 1$ ser fortemente activado retrospectivamente pelas condutas de condução da bola (CB) e de combinação táctica com progressão (CTP), mas inibir prospectivamente essas mesmas condutas. Por outro lado, o 1x1 estimula fortemente o aparecimento da conduta de remate (REM) prospectivamente, mas não é activado retrospectivamente por esta conduta.

O instrumento de obser vação utilizado pormenoriza as categorias inerentes ao $1 \mathrm{x} 1$ em três dimensões: espacialização do $1 x 1$ (zona 1, 2, 3 e 4); tipo de $1 \times 1$ (drible de progressão, de protecção, para remate e para passe); e conte xto de cooperação (sem apoios, com um apoio e com dois ou mais apoios).

A análise das subcategorias do $1 \times 1$ permitiu constatar que as características destas condutas que mais frequentemente induzem o desequilíbrio defensivo do adversário são: a ocorrência nas zonas mais ofensivas, i.e., 3 e 4 (quadro 7); a existência de dois ou mais apoios ao nível do conte xto de cooperação (quadro 8); os tipos drible de progressão (quadro 9) e drible para remate (quadro 10).

Conforme os dados do quadro 7 , prospectivamente constata-se que após o 1x1 nas zonas 3 e 4 o remate (REM), ao nível da primeira transição, foi a conduta que mais se destacou, facto que não acontecia após o 1x1 nas zonas 1 e 2 . Esta situação deve-se obviamente à proximidade destas zonas relativamente à baliza, o que leva a que os jogadores optem pelo $1 x 1$ como estratégia para criar uma situação imediata de finalização. 
Quadro 7. Análise prospectiva ao $1 \times 1$ nas zonas 3 (D3) e 4 (D4).

\begin{tabular}{|c|c|c|c|c|c|}
\hline CC & 1 & 2 & 3 & 4 & 5 \\
\hline \multirow[t]{2}{*}{ D3 } & $\begin{array}{l}\text { REM (11.47) } \\
\text { FAL (7.67) } \\
\text { IAD [7.28) } \\
\text { PLL (4.53) } \\
\operatorname{PBD}(3.96)\end{array}$ & $\begin{array}{c}\text { PBD }[5.88] \\
\text { PC }[4.06] \\
\text { CRUZ }[2.81] \\
\text { DUEL }(2.61) \\
\text { PLL }(2.48)\end{array}$ & $\cdots$ & $\cdots$ & $\cdots$ \\
\hline & $p=0.0000$ & $p=0.0000$ & $p=n s$ & $p=n s$ & $p=n s$ \\
\hline CC & 1 & 2 & 3 & 4 & 5 \\
\hline \multirow[t]{2}{*}{ D4 } & $\begin{array}{c}\text { REM }[10.82] \\
\text { CRUZ }[6.95) \\
\text { FAL }(4.74) \\
\operatorname{TP}[4.62] \\
\text { D4 }[4.11] \\
\operatorname{PLL}(3.71) \\
\operatorname{IAD}(2.05)\end{array}$ & $\begin{array}{c}\text { PC }[5.85] \\
\text { IAD }[5.48] \\
\text { PBD }[5.34] \\
\text { PLL }[4.64] \\
\text { REM }(3.48] \\
\operatorname{GOLO}(3.17) \\
\text { D4 }[2.18]\end{array}$ & $\begin{array}{l}\text { CRUZ }[6.70] \\
\text { LB }[2.99) \\
\operatorname{PC}(2.47) \\
\text { CTR }[2.44] \\
\operatorname{REM}[2.18] \\
\text { IGR }[2.15]\end{array}$ & $\cdots$ & $\begin{array}{c}\text { GOLO (2.81) } \\
\text { PLL (2.66) } \\
\text { D4 (2.20) } \\
\operatorname{REM}(2.10)\end{array}$ \\
\hline & $p=0.0000$ & $p=0.0000$ & $p=0.0000$ & $p=n s$ & $p=0.0000$ \\
\hline
\end{tabular}

Também a perda de bola directa (PBD) apresenta frequências elevadas, o que significa que nas zonas em causa existe uma elevada probabilidade do $1 \mathrm{x} 1$ activar a perda da posse da bola.

Para além do remate, também a conduta de falta apresenta uma elevada probabilidade de ocorrência logo após o $1 \times 1$ nas zonas 3 e 4, com especial incidência na primeira. Tal parece significar que o defesa, quando em contenção, ao ver-se ultrapassado nestas zonas recorre à falta, para que o atacante não consiga tirar partido do desequilíbrio criado através do $1 \times 1$.
Pode assim considerar -se que o $1 \mathrm{x} 1$ nas zonas 3 e 4 apresenta uma elevada probabilidade de provocar um acentuado desequilíbrio na estrutura defensiva, uma vez que activa situações de remate e falta. No caso específico do $1 \mathrm{x} 1$ na zona 4 salienta-se, ainda, o facto deste activar a conduta de golo ao nível da segunda e da quinta transições.

Comparativamente com o $1 \mathrm{x} 1$ com outros conte xtos de cooperação, o $1 \times 1$ com vários apoios apresenta uma probabilidade mais elevada de conduzir o ataque à conduta de remate, apresentando por isso um maior potencial de desequilíbrio para a estrutura defensiva (ver quadro 8).

Quadro 8. Análise prospectiva ao 1x1 com vários apoios (DVA].

\begin{tabular}{|c|c|c|c|c|c|}
\hline $\mathrm{CC}$ & 1 & 2 & 3 & 4 & 5 \\
\hline \multirow[t]{2}{*}{ DVA } & $\begin{array}{c}\text { REM (11.66) } \\
\text { IAD [6.45) } \\
\text { FAL [5.38] } \\
\operatorname{TP~}[3.57) \\
\text { PLL [3.32] } \\
\text { DUEL [1.97) }\end{array}$ & $\begin{array}{c}\text { PBD (7.13) } \\
\text { PC (3.38) } \\
\text { DUEL (3.03) } \\
\text { PLL (2.74) }\end{array}$ & $\begin{array}{c}\mathrm{PC}[2.81] \\
\text { CRUZ [2.58] }\end{array}$ & IGR [5.31] & $\cdots$ \\
\hline & $p=0.0000$ & $p=0.0000$ & $p=0.045310$ & $p=0.013725$ & $\mathrm{p}=\mathrm{ns}$ \\
\hline
\end{tabular}


A análise do quadro 9 leva-nos a concluir que o $1 \mathrm{x} 1$ materializado através do drible de progressão, aumenta a probabilidade de ocorrência de condutas com um carácter crítico para o jogo. Apenas ao nível da transição 2 verificámos o aparecimento da conduta combinação táctica com retrocesso.

Quadro 9. Análise prospectiva ao drible de progressão (DPROG].

\begin{tabular}{|c|c|c|c|c|c|}
\hline CC & 1 & 2 & 3 & 4 & 5 \\
\hline \multirow{6}{*}{ DPROG } & FAL (15.53) & & \multirow{5}{*}{$\begin{array}{c}\text { PC [4.52] } \\
\operatorname{REM}(3.08]\end{array}$} & \multirow{5}{*}{$\varepsilon$} & \multirow{5}{*}{$\varepsilon$} \\
\hline & $\operatorname{IAD}(12.45]$ & $\mathrm{PBD}(4.85)$ & & & \\
\hline & PLL (9.54] & CTR (4.15) & & & \\
\hline & PBD (9.42] & REM (3.53) & & & \\
\hline & DUEL (5.81) & & & & \\
\hline & $p=0.0000$ & $p=0.0000$ & $p=0.000147$ & $\mathrm{p}=\mathrm{ns}$ & $p=n s$ \\
\hline
\end{tabular}

Todas as outras condutas que obtiveram resultados significativos indicam saída da bola do terreno e/ou paragem do jogo (PLL, PC, FAL), inter venção do adversário sobre a bola (IAD), $1 \times 1$ em que nenhum dos jogadores se encontra de posse da bola (DUEL), perda da bola por parte do ataque (PBD) e situações de finalização (REM).

Por seu lado, e como seria de esperar, o drible para remate (DREM) activa situações de remate (REM) ao nível da primeira transição. Na transição 2 já é possível encontrar um número mais elevado de condutas cujo aparecimento é, possivelmente, activado pelo drible para remate (DREM). São elas, o pontapé de canto (PC), o pontapé de linha lateral (PLL), a perda de bola directa (PBD), o golo (GOLO), o duelo (DUEL) e a perda de bola indirecta (PBD). Os resultados apresentados no quadro 10 parecem indicar-nos que o drible para remate é, de todas as subcategorias, aquela que parece provocar um maior desequilíbrio da estrutura defensiva, uma vez que conduz imediatamente a acções de finalização (remate), e ao nível da segunda transição apresenta mesmo uma probabilidade significativa de conduzir à conduta de golo.

Quadro 10. Análise prospectiva ao drible para remate (DREM].

\begin{tabular}{|c|c|c|c|c|c|}
\hline CC & 1 & 2 & 3 & 4 & 5 \\
\hline \multirow[t]{2}{*}{ DREM } & REM (39.41) & $\begin{array}{c}\text { PC }[16.06] \\
\text { PLL }[7.35] \\
\text { PBD }[6.74] \\
\text { GOLO }(5.08) \\
\text { DUEL }[2.96] \\
\text { PBI }[2.26]\end{array}$ & $\begin{array}{l}\text { CTR (8.02) } \\
\text { LB [5.03] } \\
\text { CRUZ [3.95] }\end{array}$ & $\varepsilon$ & $\varepsilon$ \\
\hline & $p=0.0000$ & $p=0.0000$ & $p=0.0000$ & $p=n s$ & $p=n s$ \\
\hline
\end{tabular}

Saliente-se que o $1 x 1$ para remate, provavelmente em virtude da acção de remate que se lhe segue, apresenta uma probabilidade acima do esperado de provocar situações de interrupção temporária ou definitiva do processo ofensivo, conduzindo muitas vezes, ao nível da transição 2, a situações de pontapé de canto, pontapé de linha lateral, perda de bola directa e perda de bola indirecta.

$\mathrm{Na}$ transição 3 pode notar -se uma probabilidade significativa de ocorrência das condutas combinação táctica com retrocesso (CTR), cruzamento (CRU Z) e lançamento de baliza (LB). 


\section{CONCLUSÕES}

A partir dos resultados obtidos parece plausível retirar as seguintes conclusões:

- O maior número de situações de $1 \times 1$ verificou-se na zona intermédia ofensiva (zona 3 ), enquanto o menor número ocorreu na zona defensiva (zona 1). - O drible de progressão foi o mais utilizado, tendo tido a sua maior incidência na zona $3(45,5 \%)$, e o $1 \mathrm{x} 1$ apresentou maior frequência em contextos de cooperação em que o portador da bola dispunha de mais do que um apoio/linha de passe.

- Prospectivamente, o $1 \times 1$ parece fomentar o desequilíbrio defensivo do adversário, uma vez que é activador de situações de falta e de remate. É também frequentemente seguido por condutas de intervenção do adversário, perda de bola e saída desta do terreno de jogo, com continuação do processo ofensivo através de pontapé de linha lateral ou de canto.

- Retrospectivamente, o $1 \mathrm{x} 1$ parece ser activado por condutas de condução de bola, combinação táctica com progressão e recuperação de bola (recuperação directa por intercepção ou recuperação directa por desarme).

- À medida que o 1x1 é realizado numa zona mais ofensiva do campo, parece aumentar a probabilidade de originar um desequilíbrio na estrutura defensiva adversária.

- Os tipos drible de progressão e drible para remate são os tipos de $1 \mathrm{xl}$ que mais provocam o desequilíbrio defensivo do adversário.

- Ao nível do conte xto de cooperação, o 1x1 com vários apoios é aquele que apresenta maior probabilidade de conduzir a uma situação de remate.

\section{INDICAÇÕES PARA O TREINO DO 1X1 NO FUTSAL}

A elevada relevância conte xtual que o 1xl apresentou ao nível do processo ofensivo, torna incontornável a sugestão de ideias para o processo de treino a partir dos resultados obtidos:

- É conveniente que o jogador tome consciência de que o $1 x 1$ não se limita ao tipo drible de progressão, no qual se procura ultrapassar o adversário directo. Embora seja o mais utilizado, existem outros tipos de drible que conduzem naturalmente a diferentes produtos (drible para remate, drible para passe ou drible de protecção).
- A existência de linhas de passe não pode, por si só, desencorajar a realização do $1 \times 1$, devendo o jogador ser instruído na capacidade de realizar a «leitura do jogo », para a partir daí tomar a decisão que considerar mais adequada. O $1 \times 1$ permite em muitos contextos situacionais ganhar uma vantagem numérica ou posicional que não é viável através de uma combinação táctica.

- Impõe-se a compreensão, por parte do jogador, da relação risco/benefício que a situação de $1 \times 1$ implica, devendo este optar por tal conduta de jogo sempre que o potencial de benefício for superior ao de risco. Assim, o recurso ao $1 \mathrm{x} 1$ deve fundamentalmente ser estimulado no meio-campo ofensivo, por apresentar uma probabilidade mais elevada de conduzir a um desequilíbrio defensivo do adversário, enquanto que deve ser desaconselhado no próprio meio campo, uma vez que se trata de uma acção de jogo que implica risco de perda da posse de bola. - Os jogadores atacantes sem bola devem ser instruídos acerca da necessidade de garantirem a cobertura ofensiva ao seu portador, com vista a diminuírem o potencial de risco em caso de perda da posse da bola.

- Encorajar os jogadores que têm receio de assumir o risco de jogar o $1 x 1$, e fazer entender aos que abusam do drible que este é um meio e não um fim «Não se joga para driblar, dribla-se para jogar melhor».

\section{CORRESPONDÊNCIA}

\section{Rui Amaral}

Rua de Diu n. ${ }^{\circ} 471^{\circ}$ Direito

4445-394 Ermesinde

Portugal

ruiamaral71@oninet.pt 


\section{REFERÊNCIAS BIBLIOGRÁFICAS}

1 Abreu, A. (2002). Análise e caracterização do processo de transição da fase defensiva para a fase ofensiva, das acções de finalização em Selecções Seniores Masculinas de Futsal. Monografia de Licenciatura. Universidade do Porto: Faculdade de Ciências do Desporto e de Educação Física.

2 Allen, J. D.; Butterfly, R.; Welsch, M. A.; Wood, R. (1998). The physical and physiological value of 5-a-side soccer training to 11-a-side match play. Journal of Human Movement Studies 34: 1-11.

3 Álvarez, J.; Salillas, L.; Virón, P.; Marqueta, P. (2002). Necesidades cardiovasculares y metabólicas del fútbol sala: análisis de la competición. Apunts - Educación Física e Deportes 67: 45-51.

4 Anguera, M.; Blanco Villaseñor, A.; López, J.; Mendo, A. (2000). La metodología obser vacional en el deporte: conceptos básicos. EFDeportes.com Revista Digital [On-line]: http://www .efdeportes.com/efd24b/obs.htm [18-092000].

5 Bezerra, P. (1999). Contributo do futebol de 5 para o desenvolvimento do jovem futebolista. Horizonte 87 (XV) 21-26.

6 Caldeira, N. (2001). Estudo da relevância conte xtual das situações de 1x1 no processo ofensivo em Futebol, com recurso à Análise Sequencial. Tese de Mestrado.

Universidade do Porto: Faculdade de Ciências do Desporto e de Educação Física.

7 Canastra, F. (2002). O desenvolvimento das acções ofensivas finalizadas no Futsal. Estudo comparativo entre as selecções nacionais seniores masculinas, no torneio de qualificação para o Campeonato Mundial de Futsal Guatemala 2000 (Paços de Ferreira). Monografia de Licenciatura. Universidade do Porto: Faculdade de Ciências do Desporto e de Educação Física

8 D'Ottavio, S. (1997). Valutazione funzionale e modello di prestazione nel calcio a 5. Notiziario del Settore Técnico (FIGC) $1: 3-8$

9 Dantas, S.; Filho, F. (2002). Futsal: identificação dos perfis, genético, de aptidão física e somatotipico que caracterizam atletas masculinos, de alto rendimento, participantes no futsal adulto, no Brasil. Fitness and Performance Journal 1 (1): 28-36.

10 Duarte, V. B. (1988). Estudo do perfil antropométrico de jogadores de Futebol de Salão da cidade de Santa Maria. Universidade Federal de Santa Maria (Dissertação).

11 Facchin, C.; Seno, M; Osimani, R. (1999). Calcio a Cinque. Il manuale dell'allenatore. Milano: Edizioni Correre.

12 Fernandes, M. (2003). O desenvolvimento das sequências ofensivas finalizadas em Futsal. Estudo comparativo entre as Selecções Nacionais Seniores Masculinas de Portugal e Espanha no Campeonato da Europa de Futsal - Itália 2003. Monografia de Licenciatura. Universidade do Porto: Faculdade de Ciências do Desporto e de Educação Física.

13 Graça, A. (1995). Os comos e os quandos no ensino do jogos. In: A. Graça, J. Oliveira (Eds.) O ensino dos jogos desportivos. Universidade do Porto: Faculdade de Ciências do Desporto e de Educação Física, Centro de Estudos dos Jogos Desportivos. 27-34

14 Hoff, J.; Wisloff, U.; Engen, L.C.; Kerni, O.J.; Helgerud, J. (2002). Soccer specific aerobic endurance training. British Journal of Sports Medicine 36: 218-221.

15 Matos, C. (2002). A relação entre a qualidade do último passe e a eficácia das acções de finalização no Futsal. Estudo comparativo entre as Selecções Nacionais Seniores Masculinas de Portugal, Espanha e Brasil. Monografia de Licenciatura. Universidade do Porto: Faculdade de Ciências do Desporto e de Educação Física.

16 Mendes, I. (2002). Análise dos “Sistemas" Defensivos das Selecções de Futsal Seniores Masculinas de Portugal e Espanha. Monografia de Licenciatura. Universidade do Porto: Faculdade de Ciências do Desporto e de Educação Física.

17 Mendo, A. (1999). Obser vación y deporte In: Anguera, M. (Ed.) Observación en deporte y conducta cinésico-motriz: aplicaciones. Barcelona: Edicions de la Universitat de Barcelona, 39-69.

18 Mendo, A.; Villena, S.; García, M.; Orozco, J.; Roldán, R. (2000). Aportaciones del análisis secuencial al baloncesto: una aproximación. EFDeportes.com Revista Digital, Año 5, N. 18 [On-line]: http://www .efdeportes.com/efd18/analis.htm [17/04/2003].

19 Molina, R. (1992). Futsal: um estudo das capacidades aeróbia e anaeróbia de jogadores e das atividades em jogo. Monografia de Licenciatura. Rio Claro: Universidade Estadual Paulista.

20 Moreno, J. (2001). Análisis de los parámetros espacio y tiempo en el fútbol sala. La distancia recorrida, el ritmo y dirección del desplazamiento del jugador durante un encuentro de competición: Los casos de J. Gay (defensa), C. Marrero (cierre), J. Beto (pivote), J. Limones (ala) y J. Clavería (portero). Apunts - Educación Física e Deportes 65: 32-44.

21 Oliveira, L. (1998). Perfil de actividade do jovem jogador de Futsal/Cinco. Um estudo em atletas juvenis masculinos. Tese de Mestrado. Universidade do Porto: Faculdade de Ciências do Desporto e de Educação Física.

22 Paulis, J.; Mendo, A. (2002). Análisis diacrónico de la acción de juego en fútbol. EFDeportes.com Revista Digital [On-line]: http://www .efdeportes.com/efd49/diacro.htm [28/12/2003].

23 Paulis, J.; Mendo, A. (2002). Obser vación y anàlisis de la acción de juego en el fútbol. In J. Garganta, A. Suarez, C. Peñas (Eds.) A Investigação em Futebol. Estudos Ibéricos. Universidade do Porto: Faculdade de Ciências do Desporto e de Educação Física, 15-27.

24 Peñas, C.; Anguera, M.; Acero, M. (2002). La Acción Motriz en los Deportes de Equipo de Espacio Común y Participación Simultánea. In J. Garganta, A. Suarez, C. Peñas (Eds.) A Investigação em Futebol. Estudos Ibéricos. Universidade do Porto: Faculdade de Ciências do Desporto e de Educação Física, 79-83.

25 Platt, D.; Maxwell, A.; Horn, R.; Williams, M.; Reilly, T. (2001). Physiological and Technical Analysis of $3 \mathrm{v} 3$ and 5 v 5 Youth Football Matches. Insight 4 (4): 23-24.

26 Sampedro, J. (1997). Fútbol Sala - Las acciones del juego. Análisis metodológico de los sistemas de juego. Madrid: Editorial Gymnos.

27 Sannicandro, I. (1995). Check up della partita nel Calcio a 5. Notiziario Settore Tecnico (FIGC) 6: 17-21.

28 Santana, W. (2003). Futebol de Salão e Futsal: 70 e poucos anos de história. [On-line]: http://www .pedagogiafutsal.com.br [17/02/2004].

29 Silva Matos, C. (2002). Perfil táctico ofensivo em Futsal. Estudo exploratório da fase ofensiva entre as Selecções 
Brasileira e Espanhola do escalão sénior masculino de Futsal. Monografia de Licenciatura. Universidade do Porto: Faculdade de Ciências do Desporto e de Educação Física.

30 Silva, C. (2002). Estudo comparativo das acções ofensivas com finalização entre equipas de níveis distintos da Divisão de Elite do Futsal Português. Monografia de Licenciatura. Universidade do Porto: Faculdade de Ciências do Desporto e de Educação Física.

31 Souza, P. (2002). Validação de teste para avaliar a capacidade de tomada de decisão e o conhecimento declarativo em situações de ataque no Futsal. Dissertação de Mestrado.

Universidade Federal de Minas Gerais. Escola de Educação Física, Fisioterapia e Terapia Ocupacional. 